This item was submitted to Loughborough's Research Repository by the author.

Items in Figshare are protected by copyright, with all rights reserved, unless otherwise indicated.

\title{
A mechanical supination sprain simulator for studying ankle supination sprain kinematics
}

PLEASE CITE THE PUBLISHED VERSION

http://dx.doi.org/10.1016/j.jbiomech.2008.05.034

PUBLISHER

(C) Elsevier

VERSION

AM (Accepted Manuscript)

\section{PUBLISHER STATEMENT}

This work is made available according to the conditions of the Creative Commons Attribution-NonCommercialNoDerivatives 4.0 International (CC BY-NC-ND 4.0) licence. Full details of this licence are available at: https://creativecommons.org/licenses/by-nc-nd/4.0/

\section{LICENCE}

CC BY-NC-ND 4.0

\section{REPOSITORY RECORD}

Chan, Yue-Yan, Daniel Tik-Pui Fong, Patrick Shu-Hang Yung, Kwai-Yau Fung, and Kai-Ming Chan. 2019. "A Mechanical Supination Sprain Simulator for Studying Ankle Supination Sprain Kinematics". figshare. https://hdl.handle.net/2134/21252. 


\section{Elsevier Editorial System(tm) for Journal of Biomechanics Manuscript Draft}

Manuscript Number: BM-D-08-00024R1

Title: A mechanical supination sprain simulator for studying ankle supination sprain kinematics

Article Type: Short Communication (max 1500 words)

Section/Category:

Keywords: biomechanics, inversion platform

Corresponding Author: Professor Kai-Ming Chan,

Corresponding Author's Institution:

First Author: Yue-Yan Chan

Order of Authors: Yue-Yan Chan; Daniel Tik-Pui Fong; Patrick Shu-Hang Yung; Kwai-Yau Fung; Kai-Ming Chan

Manuscript Region of Origin: 


\section{Referee suggestion}

Prof Cheng-Kung CHENG

Email: ckcheng@bme.ym.edu.tw

Website: http://ortho.ym.edu.tw/english/t01_2.php

Dr Lars KONRADSEN,

Email: 1ako@gentoftehosp.kbhamt.dk 


\section{Conflict of interest}

Editor of Journal of Biomechanics,

REF: Submission of manuscript "A mechanical supination sprain simulator for studying ankle supination sprain kinematics"

The authors declare no financial and personal relationships with other people or organizations that could inappropriately influence this submitted work.

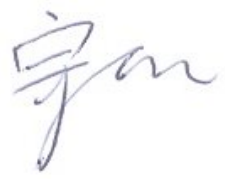

Yue-Yan CHAN

Jan 3rd, 2008. 


\section{Cover letter}

Editor of Journal of Biomechanics,

REF: Submission of manuscript "A mechanical supination sprain simulator for studying ankle supination sprain kinematics"

The authors would like to submit this paper as an "Original Article". We declare that each author were fully involved in the study and preparation of the manuscript and that the material within has not been and will not be submitted for publication elsewhere.

Corresponding author during review process: Ms. Yue-Yan CHAN, Department of Orthopaedics and Traumatology, Prince of Wales Hospital, Faculty of Medicine, The Chinese University of Hong Kong, Hong Kong, China, yychan@ort.cuhk.edu.hk

Corresponding author if paper is accepted: Prof. Kai-Ming CHAN, Department of Orthopaedics and Traumatology, Prince of Wales Hospital, Faculty of Medicine, The Chinese University of Hong Kong, Hong Kong, China, kaimingchan@cuhk.edu.hk

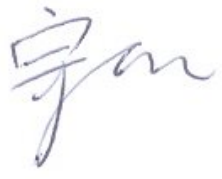

Yue-Yan CHAN

Jan 3rd, 2008. 
Journal of Biomechanics

A mechanical supination sprain simulator for studying ankle supination sprain kinematics

Yue-Yan CHAN, Daniel Tik-Pui FONG, Patrick Shu-Hang YUNG, Kwai-Yau FUNG, Kai-Ming CHAN

Decision: Request for Major Revision

\section{Response to Reviewer 1}

Reviewer 1 comments to Author...

This paper presents a mechanical apparatus for simulating ankle supination sprain injury. The authors should be congratulated on the development of this novel device which can be configured for a variety of testing scenarios. However, there are several elements of the paper which prevent me from recommending it for publication.

The paper in general would benefit from a review for readability. There are a number of errors in grammar, word choice, and sentence construct; the last sentence of the abstract is a good example of this, as I am not sure what its intended meaning is.

>> The last sentence in the original copy has contained no specific information about the sprain simulator and therefore was omitted in this version.

The introduction presents a good retrospective on ankle sprain simulators, but presents next to nothing on ankle sprain itself. It would be beneficial to see more information on why it is necessary to have combined planar motion during the sprain. As a starting point, I would suggest:

* Wright IC. Neptune RR. van den Bogert AJ. Nigg BM. The influence of foot positioning on ankle sprains. Journal of Biomechanics. 33(5):513-9, 2000 May.

* Wright IC. Neptune RR. van den Bogert AJ. Nigg BM. The effects of ankle compliance and flexibility on ankle sprains. Medicine \& Science in Sports \& Exercise. 32(2):260-5, 2000 Feb.

>> Some information about combined planarflexion and inversion motion during ankle sprain was added in line 23-26.

The methods section provides a nice description of simulator design and use. The photos could be clearer; background clutter and oblique reference frames make them difficult to interpret. A line drawing of the device would be a better choice of graphic, and would allow the inclusion of dimension information that is currently presented in text. The graphic of the solenoid is unnecessary. 


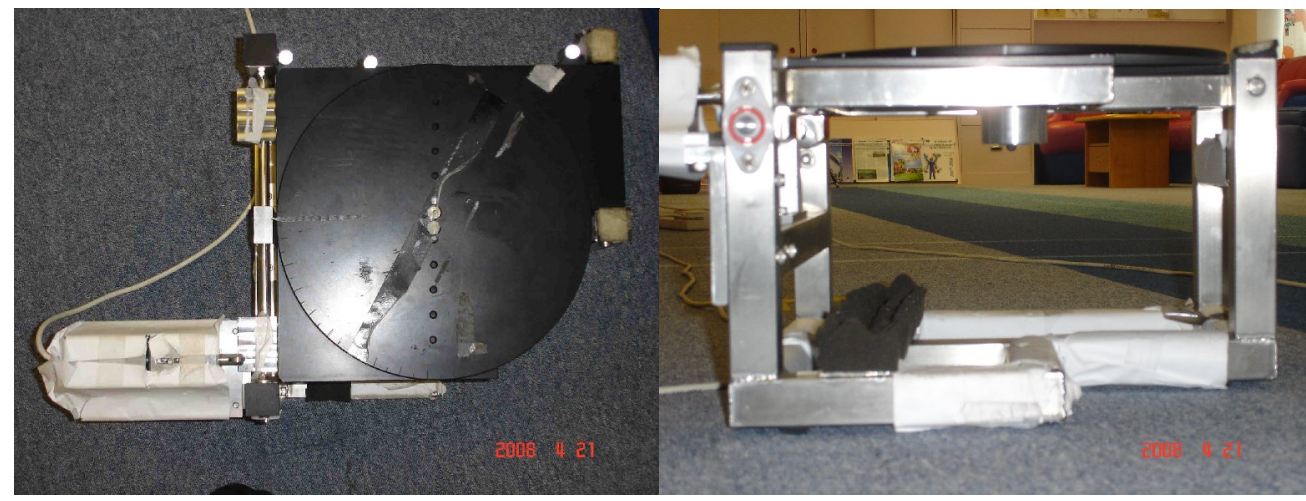

$>>$ We have taken the above 2 photos in transverse and frontal plane. However, the photos cannot clearly show the structure and measurement of the sprain simulator. Therefore, we decided to keep the original image.

I have a number of questions about the use of the device which need to be addressed in the text:

* How is the "foot axis" defined? How is the angle between the foot axis and the drop axis measured?

>> " The foot axis is defined as the line joining the heel and second metatarsal head." (line 58-59)

* How is the position of the foot on the disc standardized? Its distance from the axis of rotation will affect initial standing posture and also the biomechanics of the drop. $>>$ The position of the foot on the disc is fixed for consistency. "The foot is placed on the rotating disc with the foot axis parallel to the dotted line on the disc and the lateral edge of the foot is aligning on the dotted line." (line 59-61).

* I assume the foot slips off the surface after the trapdoor drops; how is it placed back into the same position repeatably?

$>>$ "Double-sided tape (not shown in the figure) was put on the rotating disc to prevent slippage." (line 61-62). This measures works very well by preventing slippage during the platform drop. It also allows free and natural movement of the feet at other time.

* How is disc rotation measured? Is angular rotation marked on the disc, or is a second transducer (goniometer, etc.) required?

>> "There are marks on the rotating disc, each with a 5 degrees separation, for the ease of adjustment of disc position." (line 70-72). 
* After it is rotated into position, is the disc locked into the position? What holds it in place?

>> " There is a screw beneath the platform to fix the rotating disc after adjusting to required angle." (line 49-50).

* How far does the trapdoor drop?

$>>$ In a simulated sprain, the trapdoor is released and the platform will drop for 30

degrees. "The maximum angular perturbation of the platform is 30 degrees along the rotation axis." (line 72-73).

* What safety measures are in place to prevent injury to the subject?

$>>$ "Double-sided tape (not shown in the figure) was put on the rotating disc to prevent slippage." (line 61-62). This measures works well, subjects were able to stand on the simulator safely even when the simulator on both left and right side activate at the same time.

Your methods for collecting and modeling kinematic data require more explanation. Please provide details on the specifications for the Vicon system (number of cameras, sampling rate, etc.) Also, the Vaughan ankle model requires further explanation as it is presented; at a minimum, please clarify its definition of rotation axes.

>> Specifications of Vicon system were added. "The motion was captured by a motion analysis system with 8 cameras at a rate of $120 \mathrm{~Hz}$ (VICON, USA)." (line 86-88). The details for the markers system were added. "The joint angle of the right ankle was calculated by a standard lower extremity biomechanics method (Vaughan et al., 1992), and was evaluated from one second before the trigger until one second after it. A $3 D$ reference system is formed by the location of reflective skin markers: The origin of foot segment was set at the lateral malleolus. The three markers at the right fifth metatarsal head, right heel and right lateral malleolus form the transverse plane. The internal/external rotation axis is perpendicular to this plane. The frontal-plane motion, therefore, the inversion/ eversion axis is parallel to the line joining the right heel and right fifth metatarsal head. The sagittal-plane motion, therefore the plantarflexion/dorsiflexion axis is orthogonal to the internal/external rotation and inversion/eversion axis. The steady upright anatomical standing posture was set as the offset angle in the presentation of the changes of ankle joint kinematics." (line 88-101).

I have several concerns with the results:

Did you A) use one static trial as the baseline for all trials, or B) use the early standing 
posture of each trial as its baseline? I would recommend (A); if (B), then please provide some data as to the repeatability of this standing posture.

>> Static trial was used as the baseline for all trials. "The steady upright anatomical standing posture was set as the offset angle in the presentation of the changes of ankle joint kinematics. “(line 100-101)

In your plots of ankle kinematics:

* I think your measures of inversion/eversion and internal/external rotation are reversed (mislabeled).

$>>$ Revised.

* The text at the bottom of the plot should be placed in a caption.

$>>$ Revised.

* The title "Supination Sprain" should be removed.

$>>$ Revised.

* The word "degrees" should be removed from the top row and replaced with the "o" symbol. Can you also put your foot position figures from Figure 2 up there as a visual reference?

$>>$ Revised. The two figures are combined for the ease of reading.

* You might benefit from splitting the figure into three figures - one for each plane, each with an explicative caption.

$>>$ After combining the foot position figure and the graphs into one figure, it become much clear and readable, therefore, I do not split them into three figures.

The Discussion can be expanded considerably:

* Can the relationship between increasing supination angle and increasing plantarflexion motion be explained on a anatomical level? It appears that plantarflexion motion peaks at $67^{\circ}$ supination angle, and decreased when the axis aligns with a pure plantarflexion axis. This deserves further discussion.

$>>$ The supination angle is also named as the platform angle $(\theta)$, when the platform angle increases, the rotation axis of the platform will tends to coinside to the plantarflexion/ dorsiflexion axis, therefore the plantarflexion angles increases with the platform angle. However, the variation of the position of ankle axis is great across individuals (Hertel, 2002). Therefore, the plantarflexion/dorsiflexion axis of the tested subjects is slightly oblique. From the result, the plantarflexion/dorsiflexion axis of the 
tested subjects is slightly oblique and coincide with the rotating axis of the sprain simulator when platform angle equals to 67 degrees. (line 119-124)

* I would be interested in hearing about specific applications of this device. Can you give examples of the "wide variety of sub-injury ankle sprains" to be considered? >> "Other application included study peroneal muscle function and reaction time (Konradsen and Ravn, 1991; Karlsson et al., 1992; Myers et al., 2003), or to evaluate the protective effects of braces or bracing (Cordova and Ingersoll, 2003), tape (Ricard et al., 2000) and footwear (Ottaviani et al., 1995)." (line 34-38).

* I would like an idea as to approximate cost to build in terms of parts, labor, and level of expertise required.

$>>$ "The supination sprain simulator is easy to build. It consists of an aluminium single-hinge L-shape dropping platform with rotating disc controlled by trapdoors, an aluminium L-shape supporting frame, plus solenoid operated by AC power supply. All the parts can be obtained at a low cost of about US\$500." (line 136-139). For the labor, as the structure of the sprain simulator is simple, it can be made by technician with very basic electronic and soldering skills. As the selection of labor is not that significance in the production, so, I skip it in the paper.

\section{Response to Reviewer 3}

Reviewer 3 comments to Author...

General Comments: The authors should be commended in the development of a novel device for the study of ankle joint sprain kinematics. The authors are correct to point out that no other published research methodology has described a device which allows a combination of ankle joint plantar flexion and inversion from an anatomical neutral position.

Specific comments: Starting with Page 2, with ABSTRACT being line 1.

Line 3: perhaps insert "joint" following ankle and "a" following during $>>$ Revised.

Line 4: Insert "an" following in $>>$ Revised.

Line 5: Insert "s" to make degree = degrees, insert "a" following or to make "a combination of" $>>$ Revised. 
Line 8: kinematics "were" reported instead of was reported $>>$ Revised.

Lines 11-12: Please consider something such as the following - The presented sprain simulator allows a more comprehensive study of the kinematics of ankle sprain when compared with some previous laboratory research designs.

$>>$ Revised.

Line 18: Consider replacing mechanism in with "mechanism accounting for" $>>$ Revised.

Line 19: injury should be "injuries"

$>>$ Revised.

Line 20: Consider replacing were commonly used with "have previously been used" $>>$ Revised.

Lines 21/22: Please have the references in chronological order $>>$ Revised.

Line 23: brace should be replaced by "braces or bracing" >>Revised. The sentence have been moved to line 36-37.

Line 24: Consider replacing However, most of these equipments with "However, equipment used in the aforementioned research"

$>>$ Revised.

Line 32: Please insert "neutral" after the word anatomical $>>$ Revised.

Line 32: Please consider replacing the spraining motion, with " the unexpected perturbation was induced"

$>>$ Revised.

Line: 34: Insert "an" after the word in

$>>$ Revised. The sentence changed to "This device allows the foot to perform fall of different combination of inversion and plantarflexion." 
Line 35: Insert "neutral" after the word anatomical

$>>$ Revised. The sentence was changed and the word is omitted"

Line 35: Consider including the following "the spraining motion is induced as well as allowing different combinations of inversion ..."

I would like to see a sentence about the functional or clinical relevance of the new devie and its application at the end of the introduction

$>>$ Possible application of the sprain simulator is added in line 34-38. "Other

application included study peroneal muscle function and reaction time (Konradsen

and Ravn, 1991; Karlsson et al., 1992; Myers et al., 2003), or to evaluate the protective effects of braces or bracing (Cordova and Ingersoll, 2003), tape (Ricard et al., 2000) and footwear (Ottaviani et al., 1995)."

\section{PAGE 3}

Line 42/43: This sentence does not read correctly and should read potentially as follows. "The L-shape design is to remove the front-medial corner in order to allow the close approximation of two supination sprain simulators"

$>>$ Revised.

Line 45: Replace trapdoors is at with "trapdoors are at"

$>>$ Revised.

Line 46: Insert "the" after the word at

$>>$ Revised.

Line 58: It states that the maximum angular displacement of the platform is 30 degrees. I would like the authors to clarify if this is in each plane of motion (sagittal and frontal). The device being used can induce a combination of movements. Also it would be important for the authors to discuss the potential limitations of a 30 degrees perturbation angle. Does this replicate the degree of motion that insues during an ankle sprain mechanism? For a comprehensive discussion of this issue the authors should refer to Vaes at al. Peroneal Reaction Times and Eversion Motor Response in Healthy and Unstable Ankles.J Athl Train. 2002 Dec;37(4):475-480.

>> In Osborne et al. The Effect ofAnkle Disk Training on Muscle Reaction Time in Subjects with a History of Ankle Sprain. The Americal Journal of Sports Medicine. 2001; 29(5):627-632, the perturbation angle is 20 degrees. Also, in McLoda et al. ECN. 2005; 45(1):53-58, it provide a 30 degrees inversion angle. This range of 
inversion angle was set to simulate a subinjury sprain motion. For safety and ethical reasons, the perturbation angle in our study was also set at 30 degrees. If larger perturbation angle is needed in future study, we can simply increase the height of the L-shape frame during the production of the simulator. However, safety issue should be considering in this case. (line 72-76).

Line 63: I have one reservation about the description given here and that is how the authors describe the angles of perturbation. They are labeled as follows (inversion, 23-degree supination, 45-degree supination, 67-degree supination, plantarflexion). This could be confusing considering that it states in lines 58 how "the maximum angular displacement of the platform is 30 degrees". I feel that the labeling used here is misleading and perhaps would be better labeled similar to as follows (parallel to the long axis of the foot, rotated 23 degrees relative to the long axis of the foot, etc, etc, perpendicular to the long axis of the foot).

$>>$ To make the angles more easily to read, the angle between the foot axis and rotation axis of the sprain simulator is named as the "platform angle" ( $\theta)$. Words such as "inversion", "supination" and "plantarflexion" are avoided in the angle description. For the "the maximum angular displacement of the platform is 30 degrees", it has been changed to "The maximum angular perturbation of the platform is 30 degrees" to avoid misconception.

Line 64: Please insert "the" after at to form at the lateral femoral $>>$ Revised.

Lines 64/65: The authors should clarify if this is a validated marker placement set-up. $>>$ Details of the marker set added in line 88-99. It is a validated marker set founded in Vaughan et al., 1992. A 3D reference system is formed by the location of reflective skin markers: The origin of foot segment was set at the lateral malleolus. The three markers at the right fifth metatarsal head, right heel and right lateral malleolus form the transverse plane. The internal/external rotation axis is perpendicular to this plane. The frontal-plane motion, therefore, the inversion/ eversion axis is parallel to the line joining the right heel and right fifth metatarsal head. The sagittal-plane motion, therefore the plantarflexion/dorsiflexion axis is orthogonal to the internal/external rotation and inversion/eversion axis. The steady upright anatomical standing posture was set as the offset angle in the presentation of the changes of ankle joint kinematics.

Line 69: The authors should provide the reader with information about the kinematics sampling frequency. 
>> Kinematics sampling frequency of $120 \mathrm{~Hz}$ was added in line 88-90.

Line 72: The ankle joint angle should be replaced by "The ankle joint kinematics were"

$>>$ Revised.

PAGE 4

Line 99: Please list references in chronological order $>>$ Revised.

Line 107: for the study should be replaced by "of studying" $>>$ Revised.

The readers should be given some information about the potential future application of the device for ankle sprain research purposes. What particular information will the authors gather in the future and how this will enhance the biomechanical understanding of ankle sprain injury mechanisms?

$>>$ Further application of the sprain simulator were included in the introduction part (line 34-38) and therefore not repeated in discussion. 


\begin{tabular}{|c|c|c|c|}
\hline Article type & \multicolumn{3}{|c|}{ Short Communication } \\
\hline Title & \multicolumn{3}{|c|}{$\begin{array}{l}\text { A mechanical supination sprain simulator for studying ankle } \\
\text { supination sprain kinematics }\end{array}$} \\
\hline Authors & \multicolumn{3}{|c|}{$\begin{array}{l}\text { Yue-Yan CHAN }{ }^{1,2} \text {, Daniel Tik-Pui FONG }{ }^{1,2} \text {, Patrick Shu-Hang } \\
\text { YUNG }^{1,2,3} \text {, Kwai-Yau FUNG }\end{array}$} \\
\hline Affiliations & \multicolumn{3}{|c|}{$\begin{array}{l}{ }^{1} \text { Department of Orthopaedics and Traumatology, Prince of Wales } \\
\text { Hospital, Faculty of Medicine, The Chinese University of Hong } \\
\text { Kong, Hong Kong, China. } \\
{ }^{2} \text { The Hong Kong Jockey Club Sports Medicine and Health } \\
\text { Sciences Centre, Faculty of Medicine, The Chinese University of } \\
\text { Hong Kong, Hong Kong, China. } \\
{ }^{3} \text { Gait Laboratory, Department of Orthopaedics and Traumatology, } \\
\text { Alice Ho Miu Ling Nethersole Hospital, Hong Kong, China. }\end{array}$} \\
\hline \multicolumn{4}{|c|}{ Correspondence author during review process: Yue-Yan CHAN } \\
\hline Telephone & $(852) 26321377$ & Facsimile & $(852) 26463020$ \\
\hline E-mail & \multicolumn{3}{|c|}{ yychan@ort.cuhk.edu.hk } \\
\hline \multicolumn{4}{|c|}{ Correspondence author if paper is accepted: Kai-Ming CHAN } \\
\hline Telephone & $(852) 26322728$ & Facsimile & $(852) 26463020$ \\
\hline E-mail & \multicolumn{3}{|c|}{ kaimingchan@cuhk.edu.hk } \\
\hline Total words & 1498 & Words in abstract & 135 \\
\hline Keywords & \multicolumn{3}{|c|}{ biomechanics, inversion platform } \\
\hline
\end{tabular}


ABSTRACT

This study presents a free-fall mechanical supination sprain simulator for evaluating the ankle joint kinematics during a simulated ankle supination sprain injury. The device allows the foot to be in an anatomical position before the sudden motion, and also allows different degrees of supination, or a combination of inversion and plantarflexion. Five subjects performed simulated supination sprain trials in five different supination angles. Ankle motion was captured by a motion analysis system, and the ankle kinematics were reported in plantarflexion/dorsiflexion, inversion/eversion and internal/external rotation planes. Results showed that all sprain motions were not pure single-plane motions but were accompanied by motion in other two planes, therefore, different degrees of supination were achieved. The presented sprain simulator allows a more comprehensive study of the kinematics of ankle sprain when compared with some previous laboratory research designs.

Keywords: biomechanics, inversion platform

\section{INTRODUCTION}

Ankle supination sprain is the most common injury mechanism accounting for $84 \%$ of all sport-related ankle injures (Fong et al., 2007). In order to study the kinematics of ankle supination sprain motion, trap-door or tilting inversion platforms have previously been used in various previous studies. However, equipment used in the aforementioned research introduces a pure inversion ankle motion in the human anatomical frontal plane. Since different combination of plantarflexion and inversion angles have different effect on the probability of ankle sprain to take place (Wright et al., 2000), it is essential to design a device to initiate ankle supination instead of just inversion to better study the ankle sprain kinematics. In the current literature, few similar devices, which allow two planes of motions, were designed (Vaes et al., 1998; Ricard et al., 2000). These modified inversion platforms allow the feet to be put at anatomical position to some ankle plantarflexion, thus forming a combined supination motion at subinjury level. However, in using these devices, the feet were already in plantarflexed orientations instead of anatomical neutral positions before the unexpected perturbation was induced. This paper presents a simple free-fall mechanical supination sprain simulator for studying ankle sprain kinematics in sub-injury level. Other applications of the supination sprain simulator include the study of peroneal muscle function and reaction time (Konradsen and Ravn, 1991; Karlsson et al., 1992; Myers et al., 2003), or to evaluate the protective effects of braces or bracing (Cordova and Ingersoll, 2003), tape (Ricard et al., 2000) and footwear (Ottaviani et al., 1995). As ankle is not a single hinge joint but composing of 
three articulations: the talocrural joint, the subtalar joint, and the distal tibiofibular syndesmosis, ankle sprain is not a simple one directional motion (Hertel, 2002). This device allows the foot to perform fall of different combination of inversion and plantarflexion.

\section{METHODS}

The supination sprain simulator (for right foot) is shown in Figure 1. It consists of a L-shape supporting frame which is $0.34 \mathrm{~m}$ in width and $0.25 \mathrm{~m}$ in height (Figure 2a) and a L-shape platform with a rotating disc (radius $=0.155 \mathrm{~m}$ ) on top (Figure $2 \mathrm{~b}$ ). The L-shape design is to remove the front-medial corner in order to allow the close approximation of two supination sprain simulators (Figure 3a). There is a screw beneath the platform to fix the rotating disc after adjusting to required angle. The platform is attached on the supporting frame at the rotation axis at medial edge (Figure 1). A pair of trapdoors are at the lateral edge to support the platform, which is controlled by a solenoid shutter located at the front-lateral corner (Figure 1). When an electric current passes through the solenoid, it triggers on a magnetic field to pull off the shutter that holds the trapdoors. Then the platform falls from the lateral edge to introduce a sudden ankle motion.

The standing position of the foot on the rotating disc is shown in Figure 1. The foot axis is defined as the line joining the heel and second metatarsal head. The foot is placed on the rotating disc with the foot axis parallel to the dotted line on the disc and the lateral edge of the foot is aligning on the dotted line. Double-sided tape (not shown in the figure) was put on the rotating disc to prevent slippage. We define the angle between the foot axis and the rotation axis on the sprain simulator as platform angle $(\theta)$. When platform angle equals to 0 degree, the supination sprain simulator provides a rather pure inversion motion as the foot axis is in parallel with the rotation axis (Figure 3a). If the disc is rotated clockwise, or in relative, the supporting frame and platform are rotated anti-clockwise, the foot axis starts to make an angle with the rotating axis, platform angle increases and thus a supination motion is introduced (Figure 3a). If the frame and platform are further rotated to platform angle equals to 90 degrees, the device provides a rather pure plantarflexion (Figure 3a). There are marks on the rotating disc, each with a 5 degrees separation, for the ease of adjustment of disc position. The maximum angular perturbation of the platform is 30 degrees along the rotation axis. From the literature, gross failure of anterior talofibular ligament at a mean inversion angle of $34.6 \pm 5.6$ degrees (Aydogan et al., 2006). Therefore, the 30 degrees perturbation angle allows sub-injury ankle sprain motion to be performed repetitively in a safe and ethical condition. Another supination sprain 


\section{1}

112

113

114

simulator in the opposite direction was made for the left foot.

Five male subjects (age $=23.8 \pm 2.8 \mathrm{yr}$, height $=1.72 \pm 0.05 \mathrm{~m}$, body mass $=63.7 \pm$ $9.7 \mathrm{~kg})$ performed five trials in each of the five angles ( $\theta=0$ degrees, 23 degrees, 45 degrees, 67 degrees and 90 degrees). Five reflective skin markers of $15 \mathrm{~mm}$ diameter were attached to the right lower limb at the lateral femoral epicondyle, tibial tubercle, lateral malleolus, fifth metatarsal head and heel. A static trial was captured at the beginning of the experiment, where the subject stands on the floor with a steady upright anatomical posture. This serves as the baseline for each trial. In each trial, the subject was instructed to stand on the two platforms with both feet. After the subject secured a steady upright anatomical standing posture, one of the platforms was randomly activated and dropped to trigger the sudden ankle spraining motion. The motion was captured by a motion analysis system with 8 cameras at a rate of $120 \mathrm{~Hz}$ (VICON, USA). The joint angle of the right ankle was calculated by a standard lower extremity biomechanics method (Vaughan et al., 1992), and was evaluated from one second before the trigger until one second after it. A three-dimensional reference system is formed by the location of reflective skin markers: The origin of foot segment was set at the lateral malleolus. The three markers at the right fifth metatarsal head, right heel and right lateral malleolus form the transverse plane. The internal/external rotation axis is perpendicular to this plane. The frontal-plane motion, therefore, the inversion/eversion axis is parallel to the line joining the right heel and right fifth metatarsal head. The sagittal-plane motion, therefore the plantarflexion/dorsiflexion axis is orthogonal to the internal/external rotation and inversion/eversion axis. The steady upright anatomical standing posture was set as the offset angle in the presentation of the changes of ankle joint kinematics.

\section{RESULTS}

Ankle joint angle profiles were shown in Fig 3. Zero degree represented the ankle joint position during the steady upright anatomical standing posture. The peak plantarflexion angles were reached at 0.28 to 0.29 second, ranging from 13.3 to 30.2 degrees. For inversion angle, there were two local peaks during each supination, ranging from 9.7 to 15.4 degrees at 0.14 to 0.18 second. The peak internal rotation ranged from 6.0 to 7.6 degrees at 0.12 to 0.16 second.

\section{DISCUSSION}

Results suggested that there was a general tendency of a decrease of inversion angle with the increase of platform angle. This indicated that the inversion/eversion axis of the tested subjects is coincide with the rotating axis of the sprain simulator when 
platform angle equals to 0 degree. For plantarflexion/dorsiflexion angle, there was a general tendency of increase in plantarflexion angle with the increase of platform angle from 0 degree to 67 degrees. The inversion/eversion angle decreased from 14.6 degrees to 9.9 degrees. The plantarflexion angle increased from 13.3 degrees to 30.1 degrees. However, the plantarflexion angle slightly degreased to 26.5 degrees when platform angle further increased to 90 degrees. This indicated that the plantarflexion/dorsiflexion axis of the tested subjects is slightly oblique and coincide with the rotating axis of the sprain simulator when platform angle equals to 67 degrees. However, the variation of the position of ankle axis is great across individuals (Hertel, 2002). Every sprain motion was not pure single-plane motion but was accompanied by motion in other two planes. An increase of inversion angle was accompanied by a decrease of plantarflexion angle and vice versa. However, internal/external rotation angle was kept quite constant, ranging from 6.0 to 7.6 degrees even when platform angle changed.

When the foot axis is in parallel with the rotation axis (Figure 3a), the supination sprain simulator functions in the same way as some previous design of sprain simulator (Konradsen and Ravn, 1991; Karlsson et al., 1992; Myers et al., 2003). At the same time, by adjusting of the rotating disc on the supination sprain simulator and the position of the supporting frame, the angle of supination could be adjusted. This allows a wider variety of sub-injury ankle sprain motion to be considered in kinematics studies. The supination sprain simulator is easy to build. It consists of an aluminium single-hinge L-shape dropping platform with rotating disc controlled by trapdoors, an aluminium L-shape supporting frame, plus solenoid operated by AC power supply. All the parts can be obtain at a low cost of about US\$500. Therefore, the free-fall mechanical supination sprain simulator presented in this study provides a simple but comprehensive way of studying sub-injury ankle sprain motions.

\section{ACKNOWLEDGEMENT}

The study was financially supported by the Innovation Technology Fund from the Innovation and Technology Commission, Hong Kong Special Administrative Region Government, Project Number: ITS/015/06.

\section{REFERENCES}

Aydogan, Umur, Glisson, Richard R., Nunley, James A., 2006. Extensor Retinaculum Augmentation Reinforces Anterior Talofibular Ligament Repair. Clinical Orthopaedics and Related Research, 442, 210-215.

Cordova, M.L., Ingersoll, C.D., 2003. Peroneus longus stretch reflex amplitude 
increases after ankle brace application. British Journal of Sports Medicine, $37(3), 258-262$.

Fong, D.T.P., Hong, Y., Chan, L.K., Yung, P.S.H., Chan, K.M., 2007. A systematic review on ankle injury and ankle sprain in sports. Sports Medicine, 37(1), 73-94.

Hetel, Jay, 2002. Functional anatomy, pathomechanics, and pathophysiology of lateral ankle instability, 37(4), 364-375.

Karlsson, J., Peterson, L., Andreasson, G., Hogfors, C., 1992. The unstable ankle: a combined EMG and biomechanical modeling study. International Journal of Sports Biomechanics, 8(2), 129-144.

Konradsen, L., Ravn, J.B., 1991. Prolonged peroneal reaction time in ankle instability. International Journal of Sports Medicine, 12(3), 290-292.

Myers, J.B., Riemann, B.L., Hwang, J.H., Fu, F.H., Lephart, S.M., 2003. Effect of peripheral afferent alteration of the lateral ankle ligaments on dynamic stability. American Journal of Sports Medicine, 31(4), 498-506.

Ottaviani, R.A., Ashton-Miller, J.A., Kothari, S.U., Wojtys, E.M., 1995. Basketball shoe height and the maximal muscular resistance to applied ankle inversion and eversion moments. American Journal of Sports Medicine, 23(4), 418-423.

Ricard, M.D., Schulthies, S.S., Saret, J.J., 2000. Effects of high-top and low-top shoes on ankle inversion. Journal of Athletic Training, 35(1), 38-43.

Ricard, M.D., Sherwood, S.M., Schulthies, S.S., Knight, K.L., 2000. Effects of tape and exercise on dynamic ankle inversion. Journal of Athletic Training, 35(1), 31-37.

Vaes, P.H., Duquet, W., Casteleyn, P.P., Handelberg, F., Opdecam, P., 1998. Static and dynamic roentgenographic analysis of ankle stability in braced and nonbraced stable and functionally unstable ankles. American Journal of Sports Medicine, 26(5), 692-702.

Vaughan, C.L., Davis, B.L., O'Conner, J.C. (1992). Dynamics of Human Gait:Human Kinetics Publishers Champaign.

Wright, I.C., Neptune, R.R., Bogert, A.J. van den, Nigg, B.M., 2000. The influence of foot positioning on ankle sprains. Journal of Biomechanics. 33(5), 513-519. 


\section{Figure legends}

Figure 1 - The supination sprain simulator.

Figure 2 - Parts of supination sprain simulator: (a) L-shape supporting frame, (b) L-shape platform with a rotating disc.

Figure 3 -Ankle joint angle during different type of supinaton sprain.

$\mathrm{X}$ axis: Time in second. Platform starts its motion at time $=0.0 \mathrm{~s}$.

Y axis: Ankle position in degree.

Dotted lines indicate one standard deviation from the mean. 

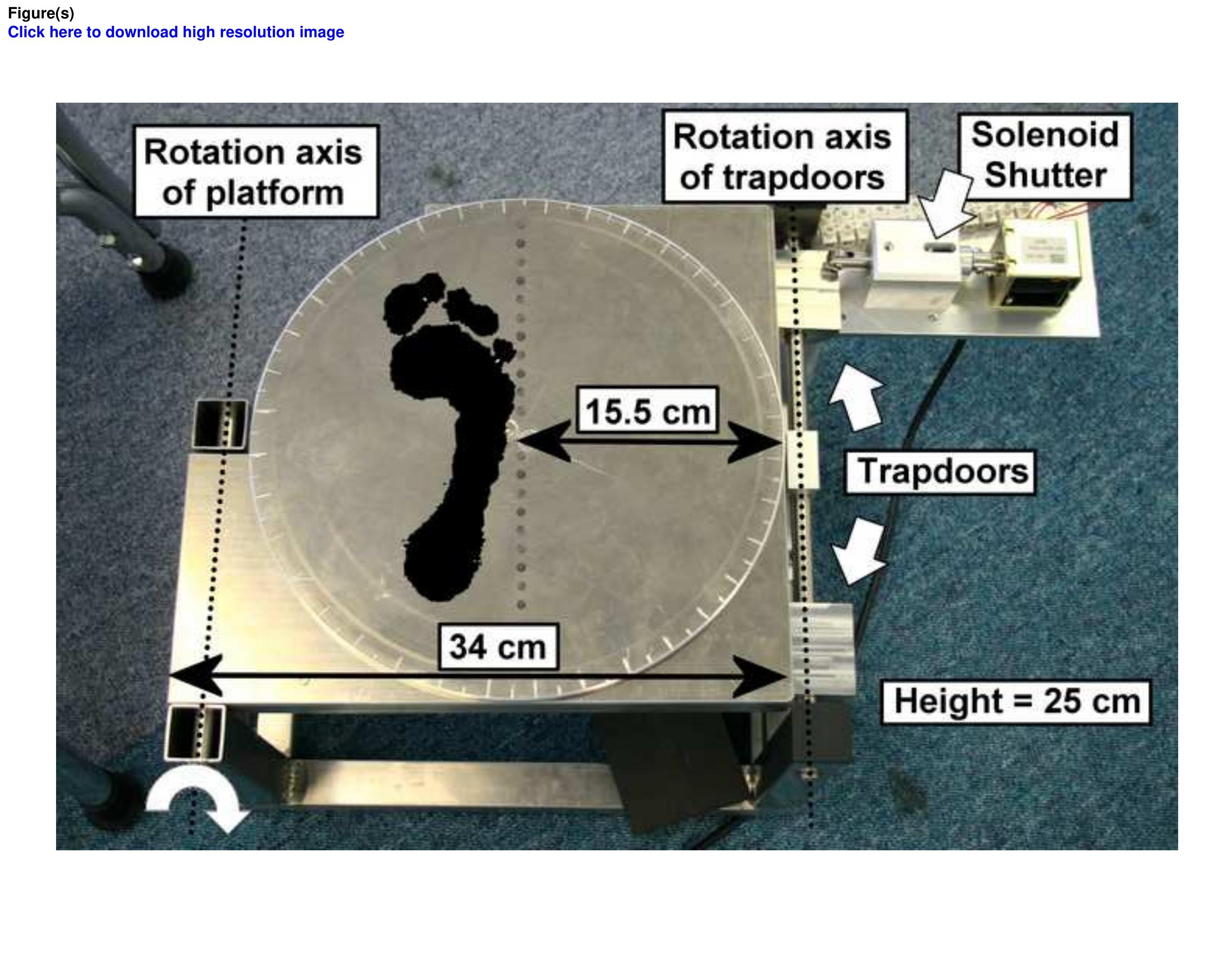

. 
Click here to download high resolution image

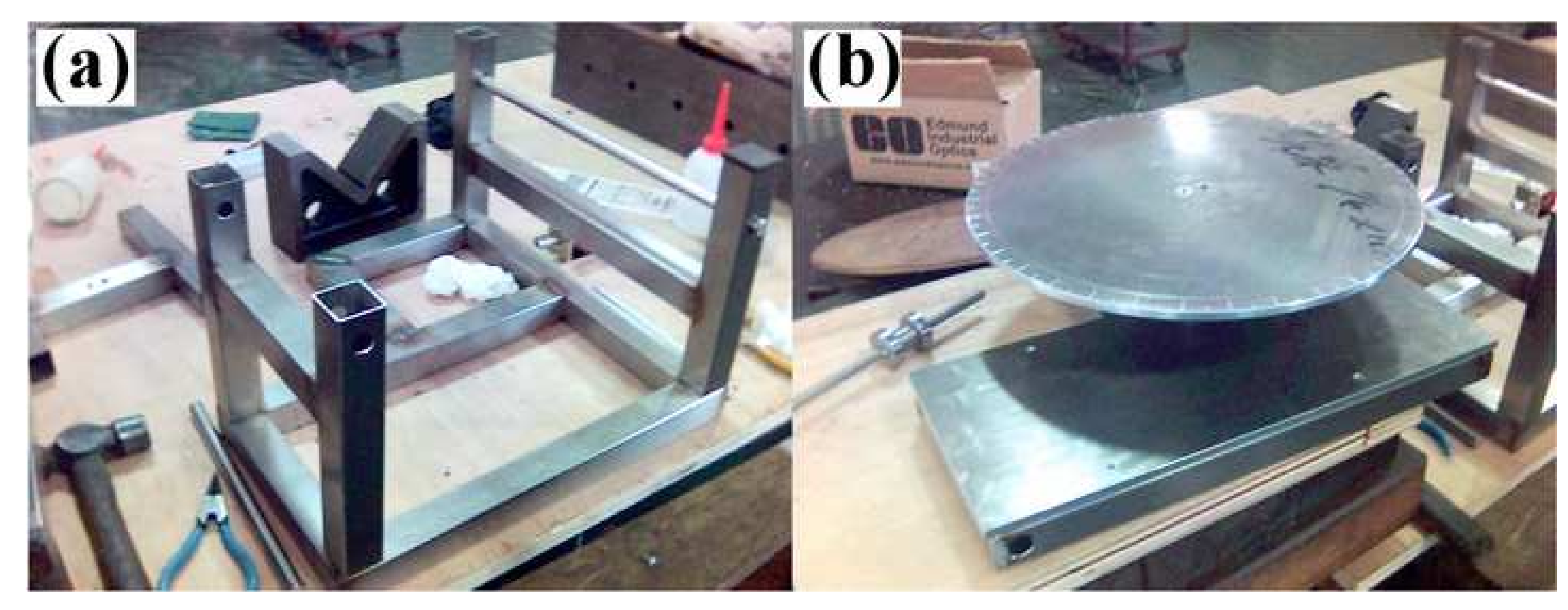




$$
\underline{\theta=0^{\circ}} \quad \underline{\theta=23^{\circ}}
$$

(a) Feet position on sprain simulator

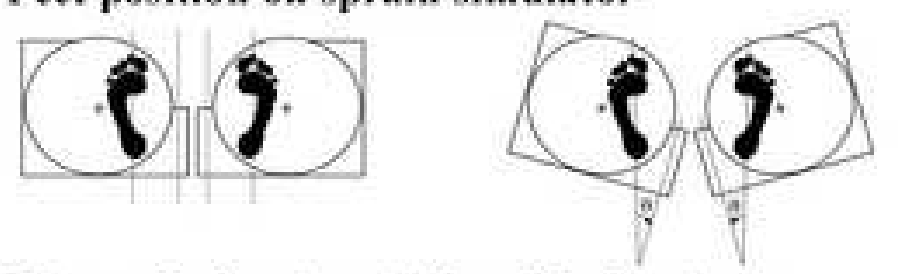

(b) Plantarflexion (+ve.) / Dorsiflexion (-ve.)
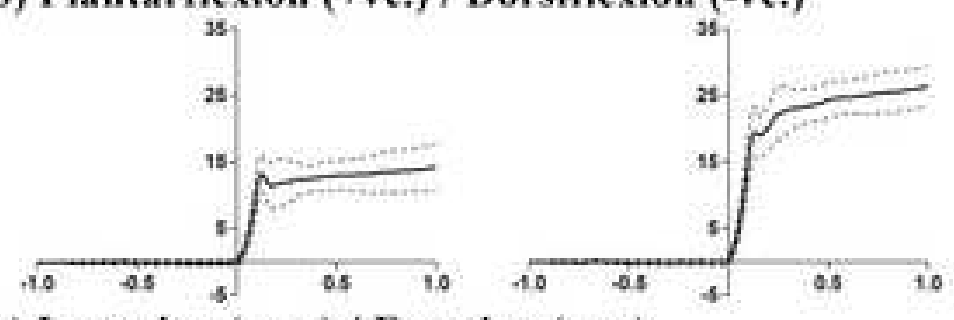

(c) Inversion (+ve.) / Eversion (-ve.)
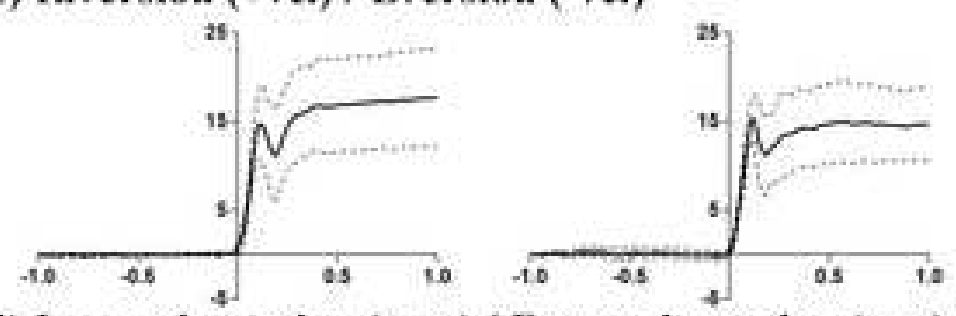

(d) Internal rotation (+ve.) / External rotation (-ve.)
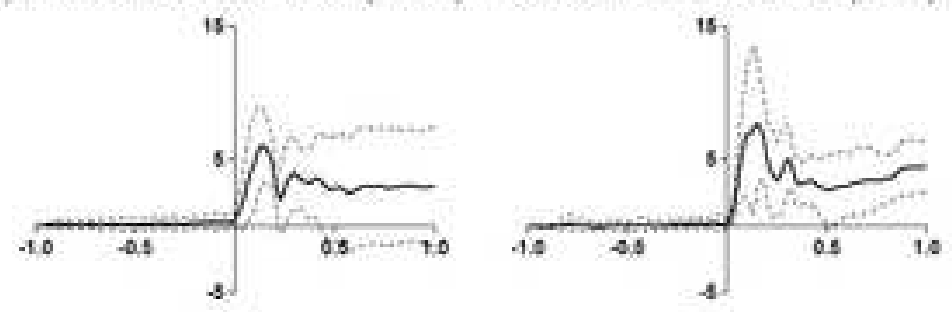

$\theta=45^{\circ}$
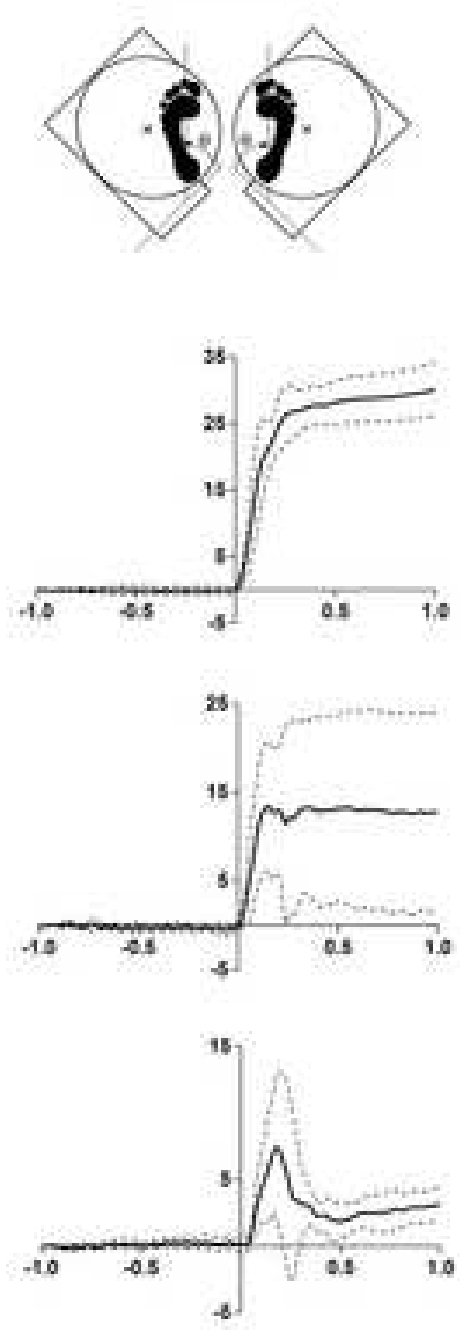

$$
\theta=67^{\circ}
$$
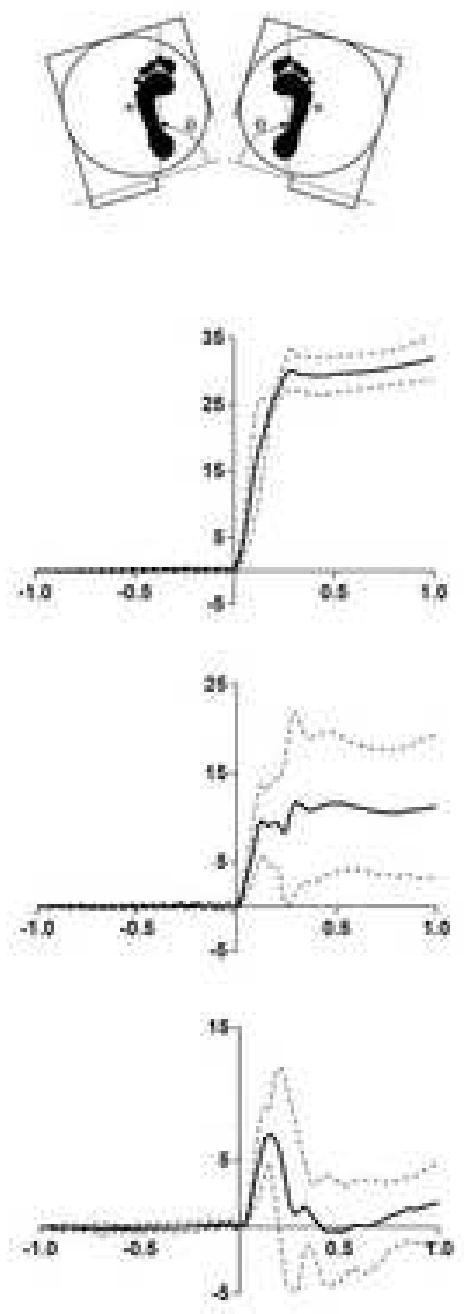

$\theta=90^{\circ}$
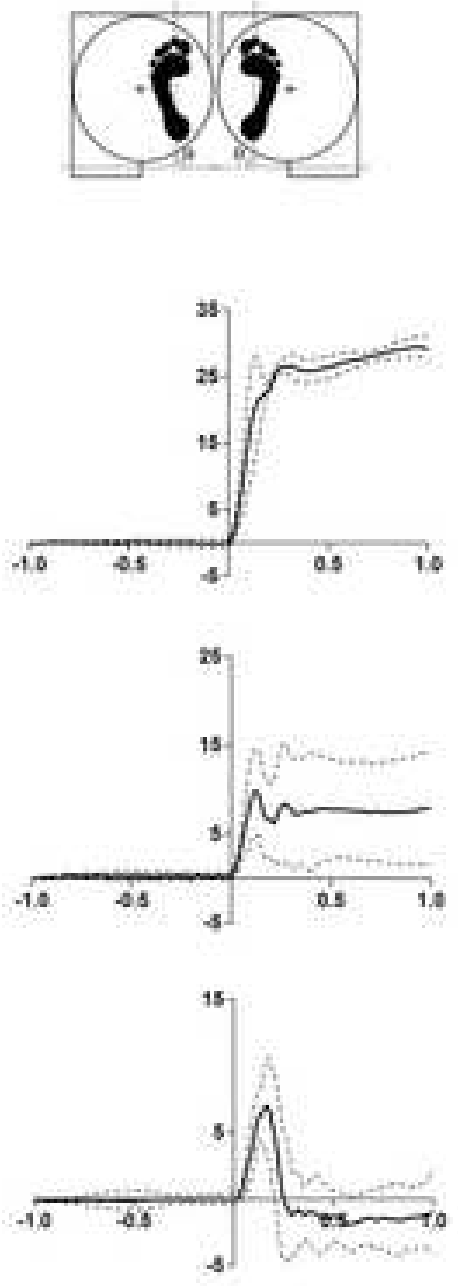